\title{
Multilayer Perceptron Learning with Particle Swarm Optimization for Well Log Data Inversion
}

\author{
Kou-Yuan Huang \\ Department of Computer Science \\ National Chiao Tung University \\ Hsinchu, Taiwan \\ kyhuang@cs.nctu.edu.tw
}

\section{Kai-Ju Chen}

National Chiao Tung University

Department of Computer Science

Hsinchu, Taiwan

chenkaiju@gmail.com

\author{
Liang-Chi Shen \\ Department of Electrical \& Computer Engineering \\ University of Houston \\ Texas, USA \\ liang.shen@gmail.com
}

\author{
Ming-Che Huang \\ National Chiao Tung University \\ Department of Computer Science \\ Hsinchu, Taiwan \\ toolwell@gmail.com
}

\begin{abstract}
Well log data inversion is important for the inversion of true formation. There exists a nonlinear mapping between the measured apparent conductivity $\left(C_{a}\right)$ and the true formation conductivity $\left(C_{t}\right)$. We adopt the multilayer perceptron (MLP) to approximate the nonlinear input-output mapping and propose the use of particle swarm optimization with mutation (MPSO) algorithm to adjust the weights in MLP. In the supervised training step, the input of the network is the measured $C_{a}$ and the desired output is the $C_{t}$. MLP with optimal size 10-9-10 is chosen as the model. We have experiment in simulation and real data application. In simulation, there are 31 sets of simulated well log data, where 25 sets are used for training, and 6 sets are used for testing. After training the MLP network, input $\mathrm{Ca}$, then $\mathrm{C}_{\mathrm{t}}$ ' can be inverted in testing process. Also we apply it to the inversion of real field well log data. The result is acceptable. It shows that the proposed MPSO algorithm in MLP weight adjustments can work on the well log data inversion.
\end{abstract}

Keywords- apparent conductivity $\left(C_{a}\right)$; true formation conductivity $\left(C_{t}\right)$; multilayer perceptron $(M L P) ;$ particle swarm optimization with mutation (MPSO);

\section{INTRODUCTION}

Well logging is a technique that uses the instruments to measure the formation information. We invert the true formation parameters from the measured information. There is a nonlinear mapping between them. Martin et al. [1] used the neural network with conjugate gradient and Levenberg-Marquardt (LM) algorithms for well log data inversion. Huang et al. [2] [3] used the higher order multilayer perceptron and radial basis function network for well log data inversion. But the methods could get the local minimum solution. Goswami et al. [4] applied the differential evolution algorithm. Szucs and Civan [5] applied the simulated annealing algorithm. But they had complex computation.

Particle swarm optimization (PSO) was proposed by Kennedy [6] that searched the global optimal solution by a population of particles distributed over the search space. It was an evolutionary technique and used cooperative mechanism to find the solution. PSO with mutation (MPSO) was proposed in [7]. The PSO algorithm had the disadvantage that it might get trapped at the local minimum if velocity was large. To solve the problem, MPSO algorithm used the mutation that the mutated particles provided a chance to escape from the local minimum.

We adopt multilayer perceptron (MLP) with MPSO algorithm in this study. MLP was able to approximate the nonlinear input-output mapping. The adjustment of weights based on the gradient descent method may get the local minimum, so we use PSO global optimization algorithm to train the weights in MLP. The supervised system of MLP with MPSO is presented in Fig. 1 for training and testing of well log data inversion.

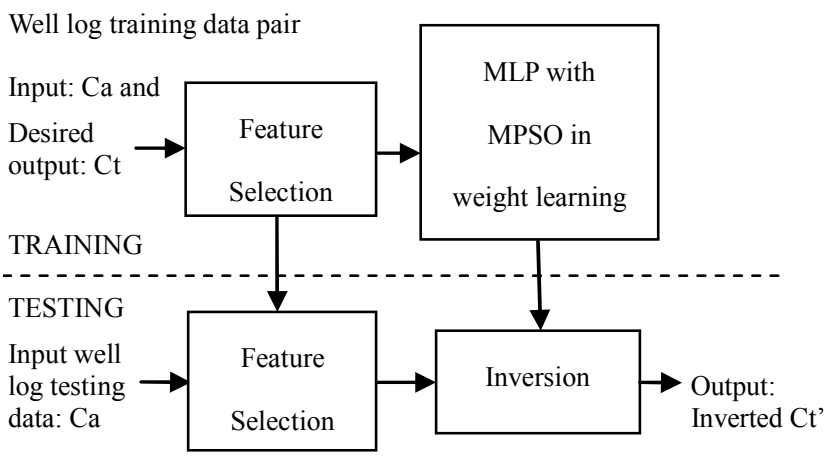

Fig. 1. System of MLP with MPSO in training and testing of well log data inversion.

Section II is the MLP weight learning with MPSO. Section III is the procedures of training and testing. Section IV is the experimental results. Finally, section V is the conclusions. 


\section{MLP WEIGHT LEARNING AND MPSO}

PSO simulates the behavior of flocks of birds [6]. Initially we set up $m$ particles. One particle in PSO is one MLP network. A two-layer MLP using MPSO learning is shown in Fig. 2. The input value of the first layer $x^{1}=$ $\left[x_{1}, x_{2}, \cdots, x_{I}, 1\right]^{\mathrm{T}}$ is $\mathrm{C}_{\mathrm{a}}$ and the output value in the output layer is the inverted $\mathrm{C}_{\mathrm{t}}$. The output value of hidden node $j$ is

$$
s_{j}=\sum_{i=1}^{I+1} w_{j i} x_{i}
$$

The output value of output node $k$ is

$$
s_{k}=\sum_{j=1}^{J+1} w_{k j} o_{j}
$$

The activation function is a sigmoidal transfer function. So, the outputs of the second layer, $o_{j}$, and the third layer, $o_{k}$, are

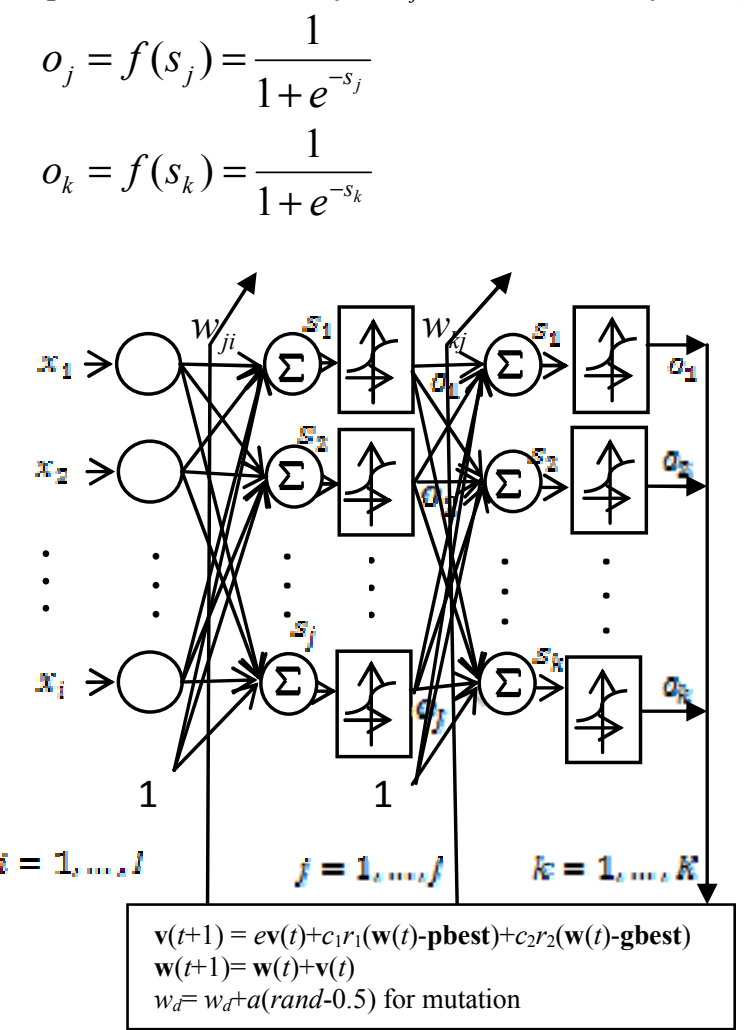

Fig. 2. Two-layer perceptron.

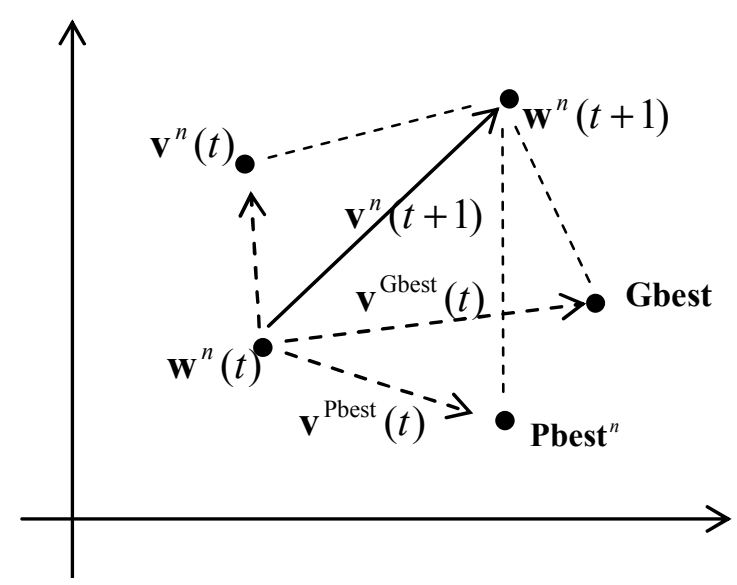

Fig. 3. The movements of a particle in MPSO.

We concatenate the two-layer weighting coefficients of MLP to be a vector as a particle in MPSO. A particle is represented as

$$
\mathbf{w}^{n}=\left[w_{l, l}, \ldots, w_{j, i}, \ldots, w_{J, I+1}, w_{l, l}, \ldots, w_{k, j}, \ldots, w_{K, J}, w_{K, J+1}\right],
$$

where $n=1, \ldots, m, n$ is the index of particle, $m$ is the number of particles, $w_{j, i}$ and $w_{k, j}$ are the weighting coefficients in MLP shown in Fig. 2. The weighting vector $\mathbf{w}$ is equivalent to position in MPSO.

Each particle has a velocity vector

$$
\mathbf{v}^{n}=\left[v_{l, 1}, \ldots, v_{j, i}, \ldots, v_{J, I+1}, v_{l, l}, \ldots, v_{k, j}, \ldots, v_{K, J}, v_{K, J+1}\right],
$$

which is also a velocity vector in MPSO. $\mathrm{N}$ is the length of $\mathbf{w}$ and $\mathbf{v}$.

Fig. 3 shows the movements of a particle in MPSO and we want to find the point with minimum fitness value in the search space [6]. Weighting vector adjustment in one MLP is equivalent to the movement of a particle in MPSO.

We have two important weighting vectors Pbest $^{n}$ and Gbest that affect the global minimum solution. Pbest ${ }^{n}$ is the best weighting coefficient vector that the particle itself has found. Gbest is the best weighting coefficient vector of all particles so far.

The velocity vector and weighting vector are adjusted by

$$
\begin{aligned}
& \mathbf{v}^{n}(t+1)=e \mathbf{v}^{n}(t)+c_{1} r_{1}\left(\mathbf{w}^{n}(t)-\text { Pbest }^{n}\right) \\
&+c_{2} r_{2}\left(\mathbf{w}^{n}(t)-\text { Gbest }\right), \\
& \mathbf{w}^{n}(t+1)=\mathbf{w}^{n}(t)+\mathbf{v}^{n}(t+1) \quad n=1, \ldots, m
\end{aligned}
$$

where $e$ is the inertia weight, $c_{1}$ and $c_{2}$ are the acceleration constants, $r_{1}$ and $r_{2}$ are random numbers uniformly distributed over $[0,1]$. In (5), the new velocity is composed of three terms. The first term is the inertia of the previous velocity. The second term is that the particle $\mathbf{w}^{n}(t)$ points toward its personal best weighting coefficient vector, Pbest $^{n}$. And the third term is that the particle $\mathbf{w}^{n}(t)$ points toward the best weighting coefficient vector of all particles so far, Gbest. Formula (6) is the weight 
vector adjustment, that the updated velocity is added to the current weighting coefficient vector $\mathbf{w}^{n}(t)$.

Initially, we have $m$ particles. Randomly generate the $n$-th particle with $\mathbf{v}^{n}(0)$ and $\mathbf{w}^{n}(0)$. And set $\mathbf{w}^{n}(0)$ to the personal best weighting coefficients Pbest $^{n}$. The fitness function is the sum of the squared error (SSE) and is defined as

$$
\mathrm{SSE}=\frac{1}{2} \sum_{q=1}^{Q} \sum_{k=1}^{K}\left(d_{q k}-o_{q k}\right)^{2}
$$

where $Q$ is the number of training samples and $K$ is the number of output nodes. $d_{q k}$ is the desired output of the $k^{\text {th }}$ output node of the $q^{\text {th }}$ training sample and $o_{q k}$ is the real output of the $k^{\text {th }}$ output node of the $q^{\text {th }}$ training sample. Each particle has a fitness value in (7). Pbest ${ }^{n}$ and Gbest use the (7) in the calculation of fitness value.

Mutation is the process to possibly lead some of the swarms away from the current position by changing the mutated particle's element. For mutation, the first step is the mutation of the particles. Ten percent of particles are selected to mutate with uniform random probability. At the second step, from mutated particle, one element $w_{d}$ with uniform random probability $1 / \mathrm{N}$ is mutated and the selected element mutates according to (8).

$$
w_{d}=w_{d}+a(\text { rand }-0.5)
$$

where $a$ is a constant that controls the amplitude of the mutated component, rand is the uniform random number distributed over $[0,1]$. Fig. 4 shows the flowchart of MPSO algorithm for weight adjustments in MLP.

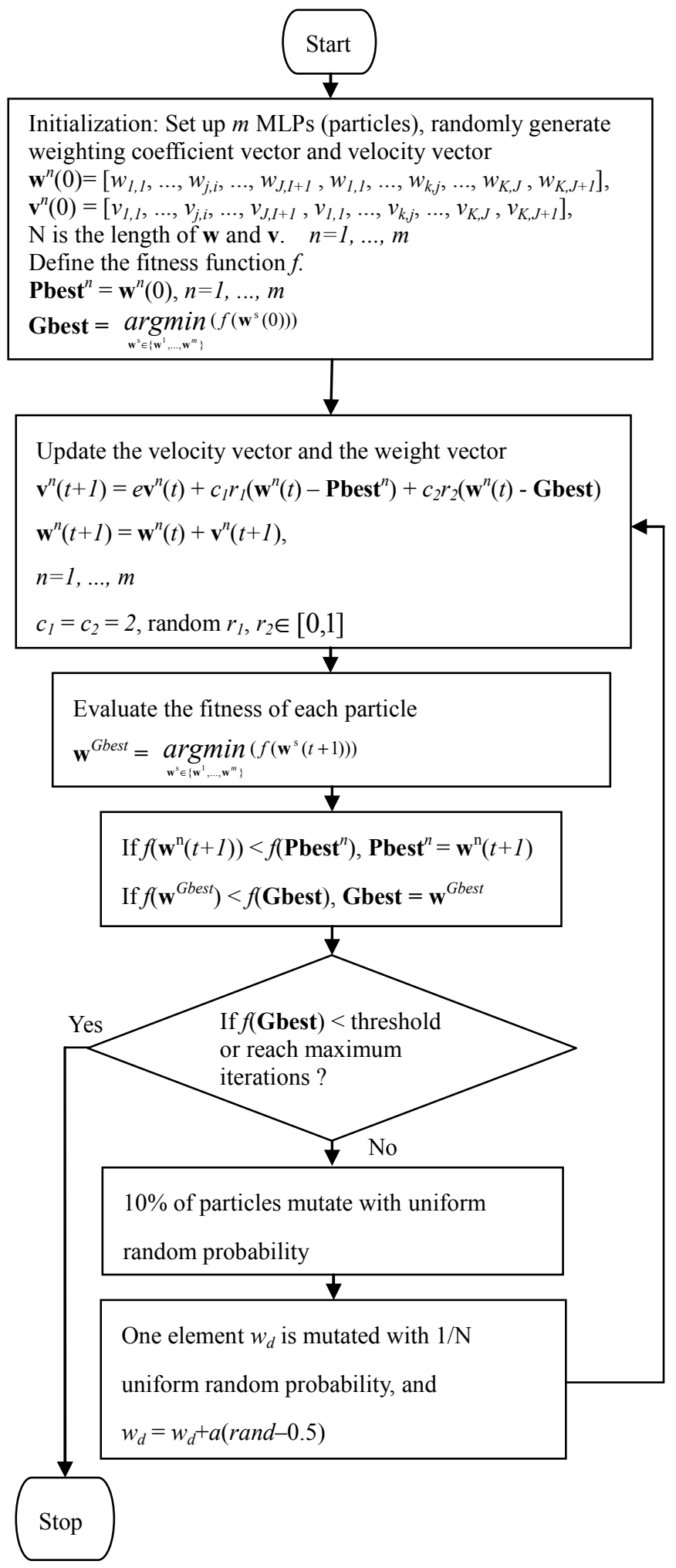

Fig. 4. Flowchart of MPSO for weight adjustments in MLP.

\section{PROCEDURES OF TRAINING AND TESTING}

We follow the procedures of training and testing in Fig. 1. There are 31 synthetic well log datasets that are calculated by the electromagnetic theory. Each simulated well log is from 
490 to 589.5 feet depth and the sample interval is 0.5 feet. Hence, there are $200 C_{a}$ and the correspondents $C_{t}$ in each simulated well $\log$. The $1^{\text {st }}$ to the $25^{\text {th }}$ simulated datasets are used for training, and others, $26^{\text {th }}$ to $31^{\text {st }}$, are used for testing.

In the training stage, we select 25 well log datasets as training where the input of the network is $\mathrm{C}_{\mathrm{a}}$ and the desired output is $\mathrm{C}_{\mathrm{t}}$. Weight adjustments are trained by MPSO algorithm. By the theorem developed by Mirchandani and $\mathrm{Cao}$ [8] and the conclusions in [2] and [3], we choose MLP with optimal size 10-9-10. In training, we take and skip every $10 C_{a}$ data as the input and every $10 \mathrm{C}_{t}$ data as the desired output. That is, when the input pattern from the $1^{\text {st }}$ to the $10^{\text {th }} \mathrm{C}_{\mathrm{a}}$ data is fed into the input layer, the next pattern fed into the input layer is the $11^{\text {th }}$ to the $20^{\text {th }} \mathrm{C}_{\mathrm{a}}$ data. Hence, for 25 well $\log$ datasets, the number of training patterns is $25 \times 200 / 10=500$.

After the convergence of training process, we adopt mean absolute error (MAE) to test 6 well log datasets from the $26^{\text {th }}$ to the $31^{\text {st }}$ well logs. The input data are every $10 \mathrm{C}_{\mathrm{a}}$, and the inverted output data are every $10 \mathrm{C}_{\mathrm{t}}$.

$$
\text { MAE }=\frac{1}{Q K} \sum_{q=1}^{Q} \sum_{k=1}^{K}\left|d_{q k}-o_{q k}\right|
$$

After the convergence of training, we also apply the MLP to the inversion of the real field well log data.

\section{EXPERIMENTAL RESULTS}

\section{A. Results of simulated well log data inversion}

We use this 10-9-10 MLP model with MPSO algorithm in the experiments. Table 1 shows the used parameters. The stop iteration is set to 20,000. Fig. 5 shows SSE of the best particle versus iteration in the training process. The SSE of the best particle after 20,000 iterations is 0.04589 . Fig. 6 shows the $26^{\text {th }}$ well $\log$ data, the dot line is $\mathrm{C}_{\mathrm{a}}$ and the solid line is $\mathrm{C}_{\mathrm{t}}$. Fig. 7 shows the $C_{a}$ and $C_{t}$ of the $31^{\text {st }}$ well $\log$ data. Fig. 8 and Fig. 9 show the inverted $C_{t}{ }^{\prime}$ and desired $C_{t}$ of the $26^{\text {th }}$ and the $31^{\text {st }}$ well logs, respectively. The dot line is the input $C_{a}$ and solid line is the inverted $\mathrm{C}_{t}{ }^{\prime}$. Table 2 shows the MAE error from the $26^{\text {th }}$ to the $31^{\text {st }}$ well logs. From the experiments, the average MAE of each point in 6 well log datasets between the inverted $\mathrm{C}_{\mathrm{t}}{ }^{\prime}$ and the desired $\mathrm{C}_{\mathrm{t}}$ is 0.004194 . It shows that MLP with MPSO gets a good result for well log data inversion. Table 3 compares the average of MAE error of 6 well log data inversion between radial basis function network (RBF) models [3] and MLP with MPSO. From the comparison, MLP with MPSO has the minimum average MAE.

Table 1. Parameters used in MLP learning with MPSO.

\begin{tabular}{cccccc}
\hline$m$ & $\mathrm{~N}$ & $e$ (decreasing linearly) & $c_{1}$ & $c_{2}$ & $a$ \\
\hline 20 & 199 & $0.9-0.4$ & 2 & 2 & 1 \\
\hline
\end{tabular}

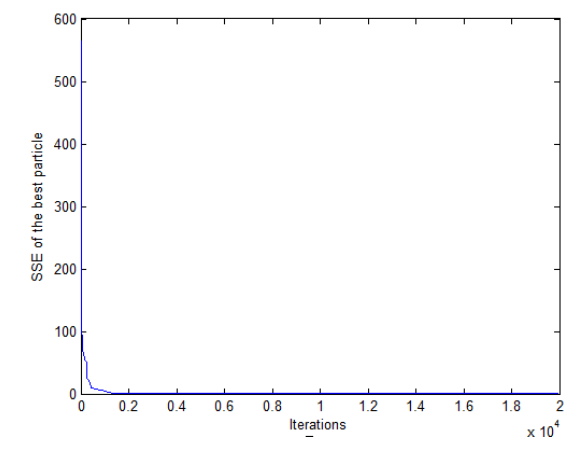

Fig. 5. SSE of the best particle vs. iteration in 10-9-10 MLP.

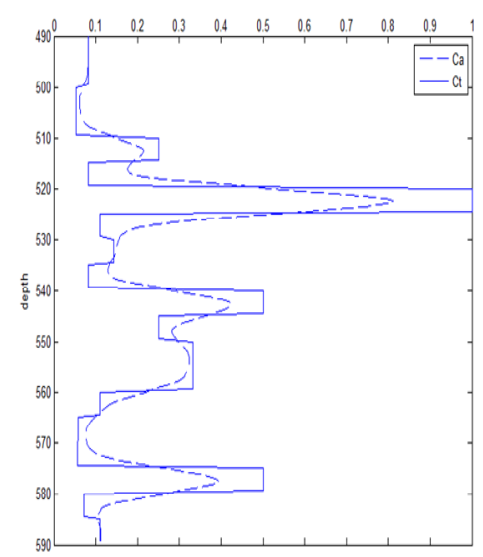

Fig. 6. Ca and $\mathrm{Ct}$ of the $26^{\text {th }}$ well $\log$.

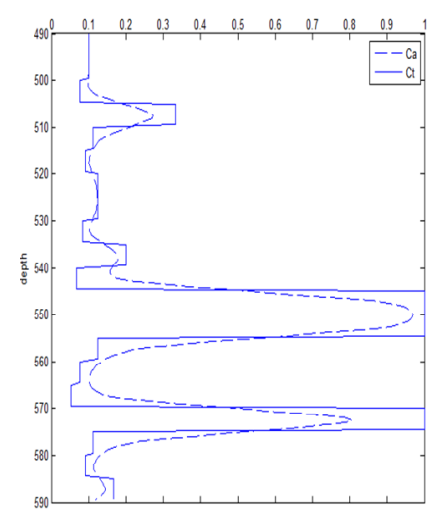

Fig. 7. Ca and Ct of the $31^{\text {st }}$ well $\log$. 


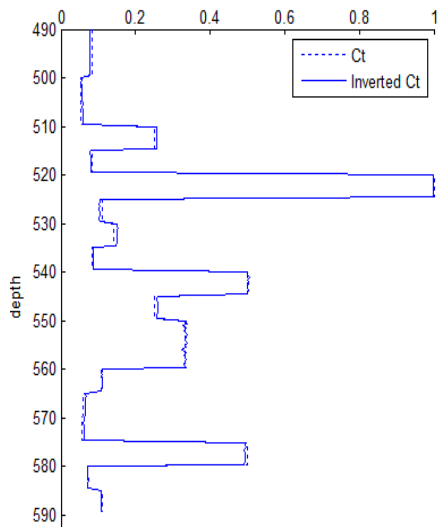

Fig. 8. Inverted $\mathrm{Ct}^{\prime}$ and desired $\mathrm{Ct}$ at the $26^{\text {th }}$ well $\log$.

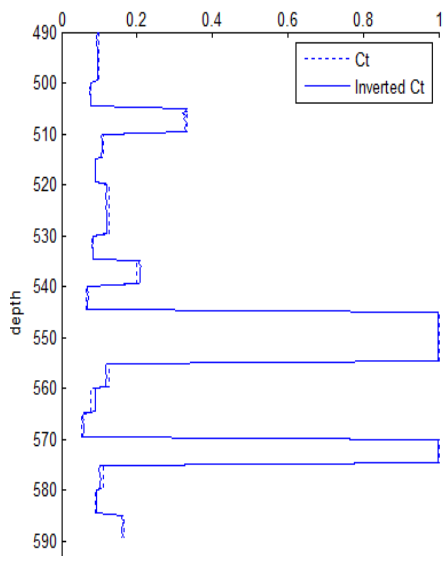

Fig. 9. Inverted $\mathrm{Ct}^{\prime}$ and desired $\mathrm{Ct}$ at the $31^{\text {st }}$ well $\log$.

Table 2. The MAE between input and inverted output from the $26^{\text {th }}$ to the $31^{\text {st }}$ well log data.

\begin{tabular}{cc}
\hline Well log data & $\begin{array}{c}\text { MAE of well log data } \\
\text { inversion }\end{array}$ \\
\hline$\# 26$ & 0.004651 \\
$\# 27$ & 0.004244 \\
$\# 28$ & 0.004574 \\
$\# 29$ & 0.003551 \\
$\# 30$ & 0.003882 \\
$\# 31$ & 0.004260 \\
\hline Average MAE & 0.004194 \\
\hline
\end{tabular}

Table 3.Comparison of average of mean absolute error of 6 well log data inversion between RBF models and MLP with MPSO.

\begin{tabular}{|c|c|c|c|}
\hline Model & $\begin{array}{c}\text { Network } \\
\text { size }\end{array}$ & $\begin{array}{c}\text { Number } \\
\text { of } \\
\text { training } \\
\text { patterns }\end{array}$ & $\begin{array}{c}\text { Average MAE of } \\
6 \text { well log data } \\
\text { inversion }\end{array}$ \\
\hline $\begin{array}{c}\text { Modified } \\
\text { two-layer RBF }\end{array}$ & $10-27-10$ & 500 & 0.048003 \\
\hline $\begin{array}{c}\text { Modified } \\
\text { three-layer RBF }\end{array}$ & $10-27-9-10$ & 500 & 0.046625 \\
\hline $\begin{array}{c}\text { MLP with } \\
\text { MPSO }\end{array}$ & $10-9-10$ & 500 & 0.004194 \\
\hline
\end{tabular}

\section{B. Results of real well log data inversion}

After the training of the 10-9-10 network, we also apply it for the inversion of real field well $\log$ data. Real field $\log$ contains 2,210 data from depth $5,577.5$ to 6,682 feet and the sample interval is 0.5 feet. Fig. 10 shows the real field data $C_{a}$. The input data to the 10-9-10 MLP is the real conductivity. Fig. 11 shows the inverted $\mathrm{C}_{\mathrm{t}}{ }^{\prime}$. Dot line is the measured real conductivity $C_{a}$ and solid line is the inverted $C_{t}$. Because there is no desired $\mathrm{C}_{\mathrm{t}}$ in real well $\log$ data, we calculate the MAE error of each point between the input $\mathrm{C}_{\mathrm{a}}$ and the inverted $\mathrm{C}_{\mathrm{t}}$ ' output, and get 0.05283 .

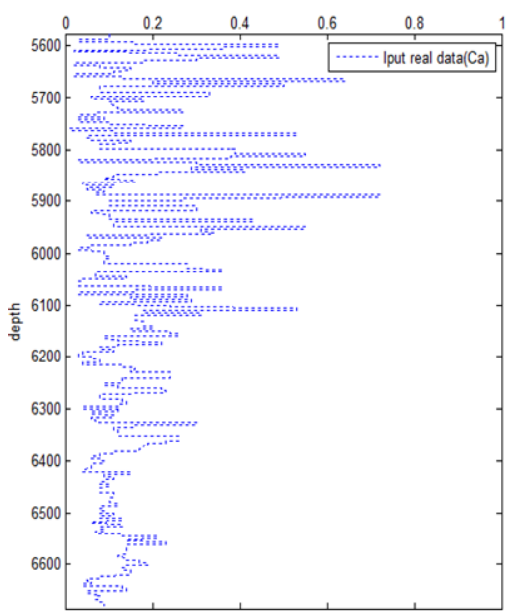

Fig. 10. Real field data with depth from 5,577.5 to 6,822 feet. 


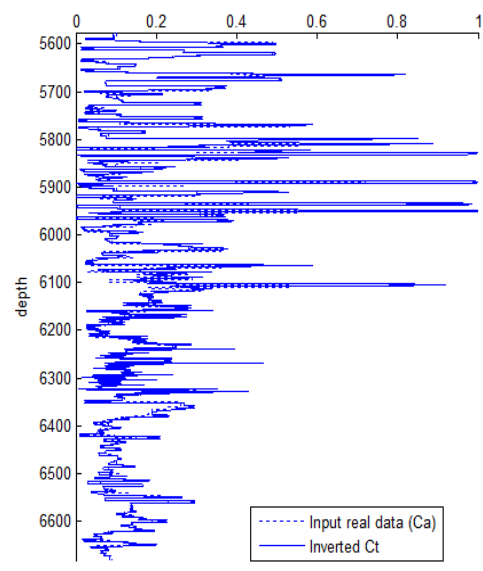

Fig. 11. Input $\mathrm{Ca}$ and inverted $\mathrm{Ct}$ ' at real data.

\section{CONCLUSIONS}

MLP with MPSO algorithm for weight training is proposed. MLP is able to approximate the nonlinear input-output mapping. The global optimization property of MPSO algorithm can avoid the local minimum during the training in MLP. In the experiments, 31 simulated well $\log$ datasets are used for supervised training and testing. The optimal network size 10-9-10 is used as the MLP model. After training of the MLP network, we apply it for the inversion of the real field well log data. The experimental result is acceptable. It shows that the proposed method can work on the well log data inversion.

\section{ACKNOWLEDGEMENT}

This work was supported in part by the National Science Council, Taiwan, under NSC100-2221-E-009-139.

\section{REFERENCES}

[1] L. S. Martin, D. Chen, T. Hagiwara, R. Strickland, G. Gianzero, and M. Hagan, "Neural network inversion of array induction logging data for dipping beds," Society of Professional Well Log Analysts, 42nd Annual Logging Symposium, Paper U, pp. 1-11, Jun 17-20, 2001.

[2] K. Y. Huang, L. C. Shen, and C. Y. Chen, "Higher Order Neural Networks for Well Log Data Inversion," IEEE International Joint Conference on Neural Networks, pp. 2545-2550, 2008.

[3] K. Y. Huang, L. C. Shen, and L. S. Wang, "Radial basis function network for well log data inversion," IEEE International Joint Conference on Neural Networks, pp. 1093-1098, 2011.

[4] J. C. Goswami, R. Mydur, P. Wu, and D. Hwliot, “A robust technique for well-log data inversion," IEEE Transactions on Antennas and Propagation, Volume 52, Issue 3, pp. 717-724, March 2004.

[5] P. Szucs and F. Civan, "Multi-layer well log interpretation using the simulated annealing method," Journal of Petroleum Science and Engineering, Volume 14, Issue 3-4, pp. 209-220, May 1996.

[6] J. Kennedy and R. C. Eberhart, "Particle Swarm Optimization," IEEE International Conference on Neural Networks, Piscataway, Volume 4, pp.1942-1948, 1995.

[7] A. Stacey, M. Jancic, and I. Grundy, "Particle swarm optimization with mutation," IEEE CEC Conference on Evolutionary Computation, Volume 2, pp. 1425-1430, 2003.

[8] G. Mirchandani and W. Cao, "On hidden nodes for neural nets," IEEE Transactions on Circuits and Systems, Volume 36, No. 5, pp. 661-664, May 1989. 